\title{
Diagnostic values of NLR and miR-141 in patients with osteoarthritis and their association with severity of knee osteoarthritis
}

\author{
CHENGCHEN CAI ${ }^{1}$, RUOJING ZHANG ${ }^{2}$, XIAOLI XU ${ }^{1}$, GUOWEN $\mathrm{LI}^{2}$ and HAIXIN GOU ${ }^{1}$ \\ ${ }^{1}$ Department of Traditional Chinese Medicine, Huadong Hospital, Fudan University, Shanghai 200040; \\ ${ }^{2}$ Department of Scientific Research, Shanghai TCM-Integrated Hospital, \\ Shanghai University of TCM, Shanghai 200082, P.R. China
}

Received July 18, 2019; Accepted July 6, 2020

DOI: $10.3892 /$ etm.2020.9506

\begin{abstract}
The present study aimed to explore the diagnostic values of neutrophil-lymphocyte ratio (NLR) and microRNA (miR)-141 in patients with osteoarthritis and their association with the severity of knee osteoarthritis. In total 142 patients with osteoarthritis (the study group) admitted to Shanghai TCM-Integrated Hospital, Shanghai University of TCM from January 2017 to January 2019 and 150 healthy controls (the control group) were enrolled in the present study. NLR and miR-141 in peripheral blood and their diagnostic values for osteoarthritis were compared between the two groups. The two indicators in the study group were significantly increased $(\mathrm{P}<0.001)$, and their combined detection had a better diagnostic value for the disease $(\mathrm{P}<0.001)$. Moreover, they were closely associated to the progression of the disease and were independent risk factors $(\mathrm{P}<0.001)$. To sum up, NLR and miR-141 were significantly increased in the peripheral blood of patients with osteoarthritis. Their combined detection exhibited a good diagnostic value for the disease and may become a potential therapeutic target osteoarthritis in the future.
\end{abstract}

\section{Introduction}

Osteoarthritis is a non-inflammatory and degenerative joint disease that is mainly characterized by arthralgia and ankylosis (1). At present, the disease mostly occurs in women, and its prevalence increases with age (2). Statistics have revealed that it affected approximately 240 million people around the world in 2016 (3), with its incidence having increased from 13.8 to $21.6 \%$ in 2018 (4). Although the cause of the disease

Correspondence to: Dr Haixin Gou, Department of Traditional Chinese Medicine, Huadong Hospital, Fudan University, 221 Yan'an West Road, Shanghai 200040, P.R. China

E-mail: g9h0jm@163.com; 17701715993@163.com

Key words: neutrophil-lymphocyte ratio, miR-141, osteoarthritis, related factors remains unclear, studies at home and abroad suggest that the cause is closely related to age and obesity, and possibly correlated with excessive joint activity, arthrotrauma, heredity, and osteoporosis $(5,6)$. Osteoarthritis mainly occurs in cartilage. The original and normal cartilage tissue undergoes morphological changes and destruction, and then forms new spurs at the articular margin (7). Moreover, the disease may also involve ligaments, joint capsules, synovium, and surrounding muscles during its course. Patients with slight conditions suffer from arthralgia and limited daily activities, while those with serious conditions suffer from joint dysfunction and paralysis $(8,9)$. Currently, osteoarthritis is diagnosed by complex joint examinations, which include erythrocyte sedimentation rate, mucin, rheumatoid factors, X-ray, CT, and MRI; however, the disease has generally progressed to the advanced stage when detected by bone scanning and arthroscopy (10).

The challenges produced by osteoarthritis have become increasingly serious, thus researchers at home and abroad are striving to find an early screening program that is more effective, in order to improve the rehabilitation effect on patients. With the advancement of research, it has been revealed that as non-coding short-stranded RNAs with a length of approximately $22 \mathrm{nt}$, microRNAs (miRs) inhibit the translation and transcription of target genes by binding to the 3' untranslated region (3'UTR) of downstream target gene mRNAs, thus altering the expression of the target gene (11). microRNAs play an important role in numerous diseases (12). It has been confirmed that microRNA-141 (miR-141) inhibits bone resorption and has aberrant expression in osteoporosis rats, which is closely related to changes in normal bone morphology $(13,14)$. However, its role in osteoarthritis remains unclear. It is theorized that this miR may be closely correlated with osteoarthritis, and thus may be particularly significant to the future diagnosis and treatment of the disease. Neutrophil-lymphocyte ratio (NLR) has a great predictive value for coronary artery disease (15), and a significant monitoring value for inflammation development (16). Therefore, miR-141 and NLR in patients with osteoarthritis were analyzed in the present study, to explore their significance and provide new insights for the clinical diagnosis and treatment of the disease. 


\section{Materials and methods}

General information. One hundred and forty-two patients with osteoarthritis (the study group) admitted to Shanghai TCM-Integrated Hospital, Shanghai University of TCM from January 2017 to January 2019 and 150 healthy controls (the control group) were enrolled in this study for a prospective analysis. The present study was approved by the Ethics Committee of Shanghai TCM-Integrated Hospital, Shanghai University of TCM. All research subjects or their immediate families signed the informed consent form.

Inclusion and exclusion criteria. Inclusion criteria were as follows: i) Patients who met clinical manifestations of knee osteoarthritis (17); ii) patients confirmed with knee osteoarthritis by a series of examinations at Shanghai TCM-Integrated Hospital, Shanghai University of TCM; iii) patients with complete medical records; iv) patients who had not received any antibiotic treatment within 3 months before admission; iv) patients willing to cooperate and participate in the investigation of medical staff at Shanghai TCM-Integrated Hospital, Shanghai University of TCM. Exclusion criteria were as follows: i) Patients complicated with tumors, cardiovascular and cerebrovascular diseases, autoimmune diseases, organ dysfunction, infectious diseases, or neurological disorders; ii) patients allergic to drugs used in the present study; iii) patients with physical disabilities and who could not take care of themselves; iv) patients who transferred to other hospitals.

Methods. After admission, the patients were orally administered loxoprofen sodium tablets (Daiichi Sankyo Pharmaceutical (Shanghai) Co., Ltd.; State Food and Drug Administration (China) Approval no. H20030769), 60 mg x3 times/day, and Gukang capsules (Guizhou Weikang Zifan Pharmaceutical Co., Ltd.; SFDA Approval no. Z20025657), $0.4 \mathrm{~g} / \mathrm{capsule}, 3$ capsules $\times 3$ times/day. The course of treatment was one month in total. Before treatment, the fasting venous blood $(5 \mathrm{ml})$ of patients was extracted and placed into EDTA anticoagulant tubes $(2 \mathrm{ml})$ and blood collection tubes $(3 \mathrm{ml})$ containing inert separation gel and coagulants. NLR expression in the venous blood of the EDTA anticoagulant tubes was detected by a flow cytometer, whereas the venous blood in the blood collection tubes was centrifuged at $1,505 \mathrm{x}$ g for $10 \mathrm{~min}$ at $24^{\circ} \mathrm{C}$, to collect the serum. Part of the serum was used for subsequent experiments, while the rest was placed in an RNase-free EP tube and stored at $-80^{\circ} \mathrm{C}$ for later use.

Reverse transcription quantitative polymerase chain reaction $(R T-q P C R)$ detection. An EasyPure miRNA kit (cat. no. ER601-01; Beijing TransGen Biotech Co., Ltd.) was used to extract total RNA from the collected serum. An ultraviolet spectrophotometer and agarose gel electrophoresis were used to detect its purity, concentration, and integrity. TransScript ${ }^{\circledR}$ miRNA RT Enzyme Mix and 2xTS miRNA Reaction Mix (cat. no. AT351-01; Beijing TransGen Biotech Co., Ltd.) were used to reversely transcribe the total RNA, with the steps in strict accordance with the manufacturer's kit. Then, PCR amplification was carried out. The system consisted of $1 \mu \mathrm{l}$ of cDNA, each $0.4 \mu \mathrm{l}$ of upstream and downstream primers, $10 \mu 1$ of TranStart ${ }^{\circledR}$ Tip Green qPCR SuperMix (cat. no. AQ141-01; Beijing TransGen Biotech Co., Ltd.), $0.4 \mu \mathrm{l}$ of Passive Reference Dye (50X) (cat. no. 600536; Beijing Biomars-Technology Co., Ltd.), and $\mathrm{dd}_{2} \mathrm{O}$ finally added to make up to $20 \mu \mathrm{l}$. The conditions were as follows: Pre-denaturation at $94^{\circ} \mathrm{C}$ for $30 \mathrm{sec}$, denaturation at $94^{\circ} \mathrm{C}$ for $5 \mathrm{sec}$, annealing and extension at $60^{\circ} \mathrm{C}$ for $30 \mathrm{sec}$, for a total of 40 cycles. Each sample was provided with 3 repeated wells, and the experiment was conducted in triplicate. In the present study, U6 was used as an internal reference and $2^{-\Delta \Delta \mathrm{Cq}}$ was used to analyze the data (18). Primer sequences are presented in Table I.

Outcome measures. Assessments included: i) Differences in miR-141 and NLR expression; ii) diagnostic values of miR-141 and NLR for osteoarthritis; iii) correlations of miR-141 and NLR with the clinical pathology of osteoarthritis; iv) correlations of miR-141 and NLR with Lysholm Knee Scoring Scale (LKSS) (19), Western Ontario and McMaster Universities Osteoarthritis Index (WOMAC) score (20), and visual analogue scale (VAS) score (21) in the study group; v) correlation of miR-141 with NLR in the study group; and iv) risk factors for osteoarthritis.

Statistical methods. SPSS 24.0 (Shanghai Yuchuang Network Technology Co., Ltd.) was used for statistical calculation. Graphpad 8 (SOFTHEAD, Inc.) was used to plot figures and verify the results. Count data including sex and dwelling environment were expressed by rate, and chi-square test was used for their comparison between groups. Measurement data for miR-141 and NLR were expressed by the mean \pm standard deviation. Independent sample t-test was used for comparison between groups, and paired t-test was used for comparison before and after treatment. One-way ANOVA and LSD post hoc test were used for the comparison among multiple groups. Receiver operating characteristic (ROC) curves were plotted to analyze the diagnostic and predictive values. Binary Logistic regression analysis was used for the combined detection, to obtain the constants and coefficients of the regression equation, and then the ROC curves were analyzed. Pearson correlation coefficient was used for correlation analysis. Multivariate Logistic regression was used to analyze risk factors. A $\mathrm{P}<0.05$, was considered to indicate a statistically significant difference.

\section{Results}

Comparison of general information. There were significant differences between the study and control groups in past medical history and exercise habits $(\mathrm{P}<0.001)$, not in age, body mass index (BMI), total cholesterol, triglyceride, low density lipoprotein, creatinine, urine nitrogen, sex, smoking, dwelling environment, and nationality $(\mathrm{P}>0.050$; Table II).

Comparison of miR-141 and NLR. The expression level of miR-141 in the study group was significantly higher than that in the control group $(17.52 \pm 4.58$ vs. $13.24 \pm 3.09)(\mathrm{P}<0.001)$. NLR in the study group $(2.81 \pm 1.14 \%)$ was significantly higher than in the control group $(1.42 \pm 0.62 \%)(\mathrm{P}<0.001$; Fig. 1$)$. 
Table I. Primer sequences of miR-141 and internal reference.

\begin{tabular}{lll}
\hline Gene & \multicolumn{1}{c}{ Forward } & Reverse \\
\hline miR-141 & 5'-CTCAAGGCAACCTACCGAAAAG-3' & 5'-TATCGGACCCATCACGGAGTGG-3' \\
U6 & 5'-GATTAGAACCGTCGGTAACGGAA-3' & 5'-AGCGATCTCGTTGGCCTTTCTACC-3'
\end{tabular}

miR, microRNA.

Table II. Comparison of general information [n (\%)].

\begin{tabular}{|c|c|c|c|c|}
\hline Characteristics & Study group $(n=142)$ & Control group $(n=150)$ & t or $\chi^{2}$ & P-value \\
\hline Age (years) & $59.4 \pm 9.1$ & $60.1 \pm 10.2$ & 0.618 & 0.537 \\
\hline $\operatorname{BMI}\left(\mathrm{kg} / \mathrm{cm}^{2}\right)$ & $23.15 \pm 1.89$ & $23.06 \pm 1.98$ & 0.397 & 0.692 \\
\hline Total cholesterol (mmol/l) & $4.42 \pm 1.20$ & $4.52 \pm 1.09$ & 0.746 & 0.456 \\
\hline Triglyceride (mmol/l) & $1.85 \pm 0.87$ & $1.90 \pm 0.77$ & 0.521 & 0.603 \\
\hline Low density lipoprotein (mmol/l) & $2.67 \pm 0.62$ & $2.58 \pm 0.72$ & 1.142 & 0.255 \\
\hline Creatinine $(\mu \mathrm{mol} / \mathrm{l})$ & $72.85 \pm 12.52$ & $71.19 \pm 11.96$ & 1.159 & 0.248 \\
\hline Urea nitrogen $(\mathrm{mmol} / \mathrm{l})$ & $6.24 \pm 1.63$ & $6.42 \pm 1.82$ & 0.889 & 0.375 \\
\hline Course of disease (years) & $3.54 \pm 1.54$ & & & \\
\hline Sex & & & 0.070 & 0.791 \\
\hline Male & $35(24.65)$ & $39(26.00)$ & & \\
\hline Female & $107(75.35)$ & $111(74.00)$ & & \\
\hline Past medical history & & & 49.172 & $<0.001$ \\
\hline Diabetes & $56(39.44)$ & $24(16.00)$ & & \\
\hline Hypertension & $42(29.58)$ & $18(12.00)$ & & \\
\hline None & $44(30.99)$ & $108(72.00)$ & & \\
\hline Smoking & & & 0.024 & 0.876 \\
\hline Yes & $48(33.80)$ & $52(34.67)$ & & \\
\hline No & $94(66.20)$ & $98(65.33)$ & & \\
\hline Dwelling environment & & & 1.417 & 0.233 \\
\hline City & $92(64.79)$ & $87(58.00)$ & & \\
\hline Countryside & $50(35.21)$ & $63(42.00)$ & & \\
\hline Nationality & & & 0.372 & 0.798 \\
\hline Han & $138(97.18)$ & $148(98.67)$ & & \\
\hline Ethnic minorities & $4(2.82)$ & $2(1.33)$ & & \\
\hline Exercise habits & & & 42.642 & $<0.001$ \\
\hline Yes & $16(11.27)$ & $69(46.00)$ & & \\
\hline No & $126(88.73)$ & $81(54.00)$ & & \\
\hline \multicolumn{5}{|l|}{ Severity } \\
\hline Early stage & $38(26.76)$ & & & \\
\hline Middle and advanced stages & $104(86.62)$ & & & \\
\hline \multicolumn{5}{|l|}{ Classification } \\
\hline Primary & $104(73.24)$ & & & \\
\hline Secondary & $38(26.76)$ & & & \\
\hline
\end{tabular}

BMI, body mass index.

Diagnostic values of miR-141 and NLR. According to the ROC curves, when the cut-off value was 14.85 , the sensitivity and specificity of miR-141 for osteoarthritis diagnosis were 72.00 and $69.01 \%$, respectively. When the cut-off value was 2.51 , the sensitivity and specificity of NLR were 96.67 and $67.61 \%$, respectively. With miR-141 and NLR as independent variables, binary logistic regression analysis was performed to obtain the logistic 

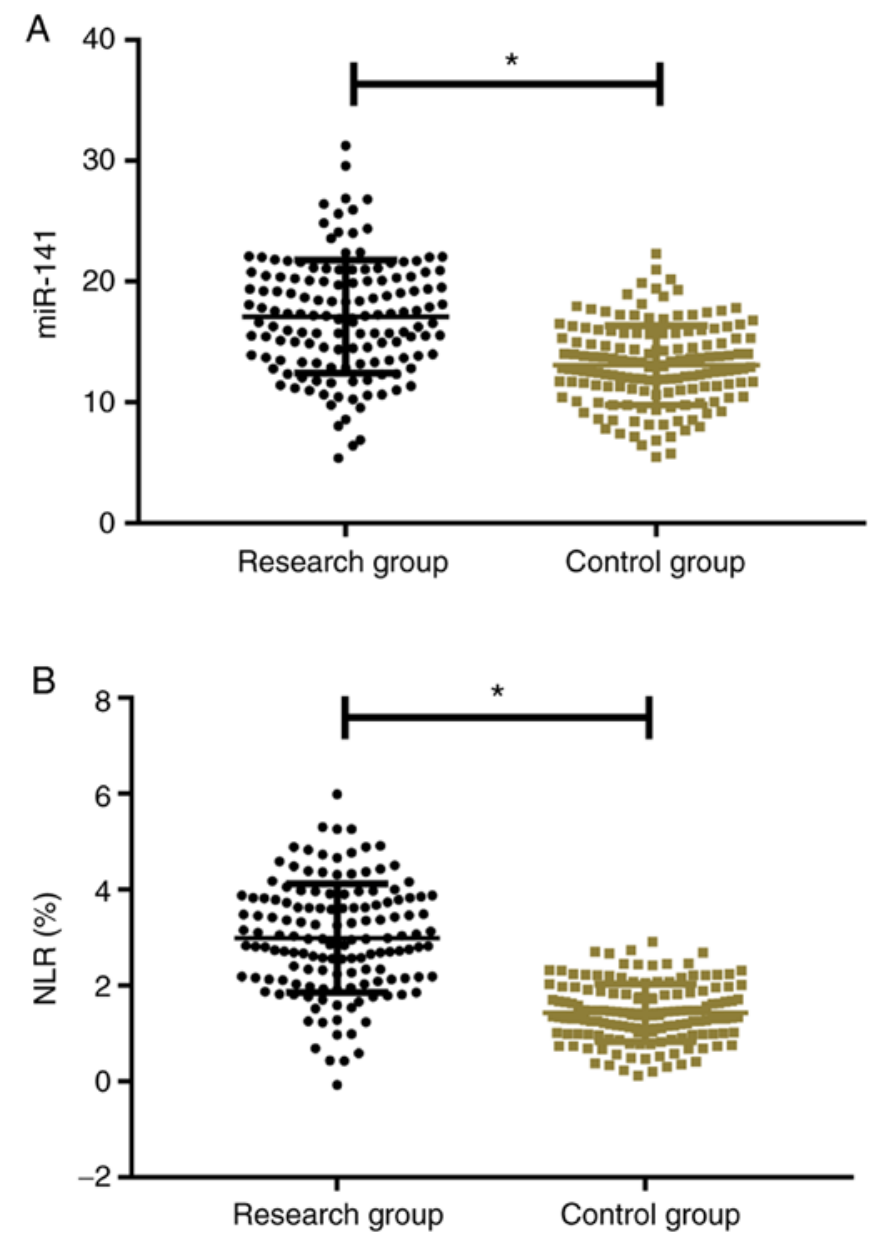

Figure 1. Comparison of miR-141 and NLR. (A) The expression level of miR-141 in the study group was significantly higher than that in the control group. ${ }^{*} \mathrm{P}<0.001$. (B) NLR in the study group was significantly higher than that in the control group. " $\mathrm{P}<0.001$. miR, microRNA; NLR, neutrophil-lymphocyte ratio.

regression model: Logit $(\mathrm{P})=-8.688+0.288 \mathrm{miR}-141+2.088$ NLR. When the cut-off value was 0.63 , the sensitivity and specificity of the model were 95.33 and $78.87 \%$, respectively (Tables III and IV and Fig. 2).

Clinicopathological associations between miR-141, NLR and osteoarthritis. miR-141 was closely associated to the course of disease, severity, and classification of diseases of patients with osteoarthritis $(\mathrm{P}<0.001)$, not significantly associated with their age, BMI, total cholesterol, triglyceride, low density lipoprotein, creatinine, urine nitrogen, sex, past medical history, smoking, dwelling environment, nationality, and exercise habits $(\mathrm{P}>0.05)$. NLR was closely associated to the age, course of disease, and severity of patients with osteoarthritis $(\mathrm{P}<0.001)$, not significantly associated with their BMI, total cholesterol, triglyceride, low density lipoprotein, creatinine, urine nitrogen, sex, past medical history, smoking, dwelling environment, nationality, exercise habits, and classification of diseases $(\mathrm{P}>0.05$; Table $\mathrm{V})$.

Correlations of miR-141 and NLR with LKSS, WOMAC, and VAS scores. Before treatment, LKSS, WOMAC, and VAS scores in the study group were $(52.62 \pm 6.21),(63.35 \pm 7.21)$, and $(8.26 \pm 2.62)$ points, respectively. According to the
Table III. Analysis of ROC curves.

\begin{tabular}{lccc}
\hline Parameters & miR-141 & NLR & miR-141+NLR \\
\hline Cut-off value & 14.85 & 2.51 & 0.63 \\
AUC & 0.753 & 0.887 & 0.927 \\
SE & 0.029 & 0.020 & 0.016 \\
$95 \%$ CI & $0.697-0.809$ & $0.847-0.927$ & $0.895-0.958$ \\
P-value & $<0.001$ & $<0.001$ & $<0.001$ \\
Sensitivity (\%) & 72.00 & 96.67 & 95.33 \\
Specificity (\%) & 69.01 & 67.61 & 78.87 \\
\hline
\end{tabular}

ROC, receiver operating characteristic; miR, microRNA; NLR, neutrophil-lymphocyte ratio; AUC, area under the curve; SE, standard error; CI, confidence interval.

Table IV. Binary logistic regression analysis.

\begin{tabular}{lcccccc}
\hline Factors & B & SE & Wald & Df & Sig & Exp (B) \\
\hline miR-141 & 0.288 & 0.051 & 32.195 & 1 & $\mathrm{P}<0.001$ & 1.334 \\
NLR & 2.088 & 0.262 & 63.571 & 1 & $\mathrm{P}<0.001$ & 8.070 \\
\hline
\end{tabular}

miR, microRNA; NLR, neutrophil-lymphocyte ratio; B, regression coefficient; SE, standard error of regression; Wald, Wald statistics; df, degrees of freedom; Sig, P-value; Exp (B), exponentiation of the $\mathrm{B}$ coefficient, which is an odds ratio.

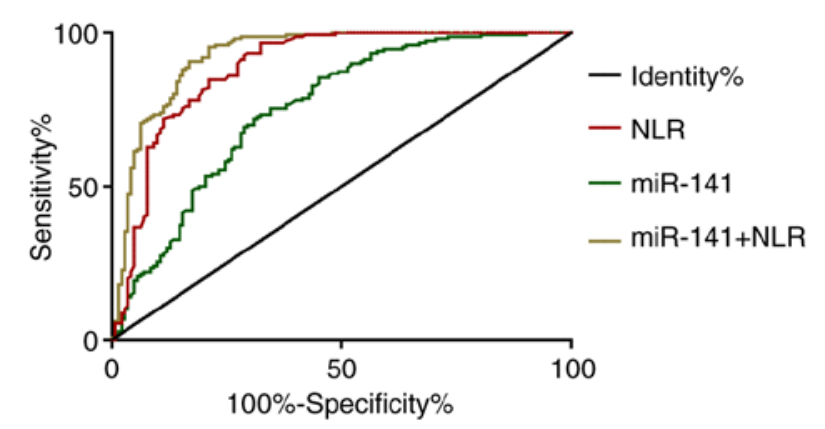

Figure 2. ROC curves of miR-141 and NLR. ROC, receiver operating characteristic; miR, microRNA; NLR, neutrophil-lymphocyte ratio.

analysis with Pearson correlation coefficient, miR-141 was negatively correlated with LKSS score $(\mathrm{r}=-0.671, \mathrm{P}<0.001)$, positively correlated with WOMAC score $(r=0.778$, $\mathrm{P}<0.001)$, and positively correlated with VAS score $(\mathrm{r}=0.846$, $\mathrm{P}<0.001)$. NLR was negatively correlated with LKSS score $(\mathrm{r}=-0.649, \mathrm{P}<0.001)$, positively correlated with WOMAC score $(\mathrm{r}=0.722, \mathrm{P}<0.001)$, and positively correlated with VAS score $(r=0.825, \mathrm{P}<0.001)$. In the study group, miR-141 was positively correlated with NLR $(r=0.889, \mathrm{P}<0.001)$ (Fig. 3).

Analysis of related risk factors. With the comparison of general information between the two groups as the results of univariate analysis, the indicators with differences were assigned values (Table VI). Then, forward logistic regression 
Table V. Clinicopathological associations between miR-141, NLR and osteoarthritis.

\begin{tabular}{|c|c|c|c|c|c|c|c|}
\hline Characteristics & $\mathrm{n}$ & miR-141 & $\mathrm{t}$ or F-value & P-value & NLR & $\mathrm{t}$ or F-value & P-value \\
\hline Age (years) & & & 0.239 & 0.811 & & 4.881 & $<0.001$ \\
\hline$<59.4$ & 42 & $17.34 \pm 4.14$ & & & $2.12 \pm 0.98$ & & \\
\hline$\geq 59.4$ & 100 & $17.55 \pm 5.01$ & & & $3.09 \pm 1.12$ & & \\
\hline BMI $\left(\mathrm{kg} / \mathrm{cm}^{2}\right)$ & & & 0.408 & 0.684 & & 0.245 & 0.807 \\
\hline$<23.15$ & 38 & $17.73 \pm 4.66$ & & & $2.92 \pm 0.84$ & & \\
\hline$\geq 23.15$ & 104 & $18.14 \pm 5.52$ & & & $2.97 \pm 1.15$ & & \\
\hline Total cholesterol (mmol/l) & & & 0.666 & 0.506 & & 1.034 & 0.303 \\
\hline$<4.42$ & 80 & $17.45 \pm 3.63$ & & & $2.71 \pm 0.92$ & & \\
\hline$\geq 4.42$ & 62 & $17.88 \pm 4.04$ & & & $2.90 \pm 1.27$ & & \\
\hline Triglyceride (mmol/l) & & & 0.194 & 0.846 & & 0.167 & 0.868 \\
\hline$<1.85$ & 83 & $17.85 \pm 3.75$ & & & $2.71 \pm 0.98$ & & \\
\hline$\geq 1.85$ & 59 & $17.72 \pm 4.17$ & & & $2.68 \pm 1.16$ & & \\
\hline Low density lipoprotein (mmol/l) & & & 0.186 & 0.852 & & 1.719 & 0.088 \\
\hline$<2.67$ & 79 & $17.57 \pm 4.87$ & & & $2.99 \pm 1.12$ & & \\
\hline$\geq 2.67$ & 63 & $17.72 \pm 4.63$ & & & $2.58 \pm 1.71$ & & \\
\hline Creatinine $(\mu \mathrm{mol} / \mathrm{l})$ & & & 0.519 & 0.605 & & 1.112 & 0.268 \\
\hline$<72.85$ & 68 & $17.14 \pm 4.59$ & & & $3.13 \pm 1.43$ & & \\
\hline$\geq 72.85$ & 74 & $17.58 \pm 5.43$ & & & $2.86 \pm 1.46$ & & \\
\hline Urea nitrogen $(\mathrm{mmol} / \mathrm{l})$ & & & 0.180 & 0.857 & & 1.131 & 0.260 \\
\hline$<6.24$ & 70 & $17.59 \pm 4.72$ & & & $2.75 \pm 1.10$ & & \\
\hline$\geq 6.24$ & 72 & $17.73 \pm 4.54$ & & & $2.52 \pm 1.31$ & & \\
\hline Course of disease (years) & & & 7.216 & $<0.001$ & & 5.227 & $<0.001$ \\
\hline$<3.54$ & 49 & $14.57 \pm 5.63$ & & & $2.50 \pm 1.24$ & & \\
\hline$\geq 3.54$ & 93 & $20.24 \pm 3.69$ & & & $3.62 \pm 1.20$ & & \\
\hline Sex & & & 0.164 & 0.870 & & 0.612 & 0.542 \\
\hline Male & 35 & $17.85 \pm 3.75$ & & & $2.80 \pm 1.24$ & & \\
\hline Female & 107 & $17.72 \pm 4.17$ & & & $2.92 \pm 0.92$ & & \\
\hline Past medical history & & & 0.756 & 0.472 & & 10.332 & $<0.001$ \\
\hline Diabetes & 56 & $17.73 \pm 4.54$ & & & $2.85 \pm 1.11$ & & \\
\hline Hypertension & 42 & $17.59 \pm 4.58$ & & & $2.72 \pm 0.92$ & & \\
\hline None & 44 & $18.62 \pm 3.64$ & & & $2.04 \pm 0.62$ & & \\
\hline Smoking & & & 0.281 & 0.779 & & 0.559 & 0.577 \\
\hline Yes & 48 & $18.08 \pm 3.95$ & & & $2.98 \pm 1.14$ & & \\
\hline No & 94 & $17.86 \pm 4.62$ & & & $3.12 \pm 1.53$ & & \\
\hline Dwelling environment & & & 0.024 & 0.981 & & 0.779 & 0.437 \\
\hline City & 92 & $17.16 \pm 4.59$ & & & $3.12 \pm 1.20$ & & \\
\hline Countryside & 50 & $17.14 \pm 4.86$ & & & $2.96 \pm 1.11$ & & \\
\hline Nationality & & & 0.080 & 0.936 & & 0.138 & 0.890 \\
\hline Han & 138 & $16.93 \pm 4.68$ & & & $2.98 \pm 1.14$ & & \\
\hline Ethnic minorities & 4 & $17.12 \pm 4.25$ & & & $3.06 \pm 1.13$ & & \\
\hline Exercise habits & & & 0.188 & 0.851 & & 0.408 & 0.684 \\
\hline Yes & 16 & $17.63 \pm 4.58$ & & & $2.85 \pm 1.24$ & & \\
\hline No & 126 & $17.86 \pm 4.62$ & & & $2.71 \pm 1.30$ & & \\
\hline Severity & & & 4.152 & $<0.001$ & & 3.628 & $<0.001$ \\
\hline Early stage & 19 & $14.62 \pm 5.86$ & & & $2.12 \pm 1.63$ & & \\
\hline Middle and advanced stages & 123 & $21.21 \pm 6.52$ & & & $3.25 \pm 1.20$ & & \\
\hline Classification & & & 12.321 & $<0.001$ & & 0.419 & 0.676 \\
\hline Primary & 104 & $15.12 \pm 2.85$ & & & $2.75 \pm 1.31$ & & \\
\hline Secondary & 38 & $22.62 \pm 4.05$ & & & $2.85 \pm 1.10$ & & \\
\hline
\end{tabular}

miR, microRNA; NLR, neutrophil-lymphocyte ratio; BMI, body mass index. 

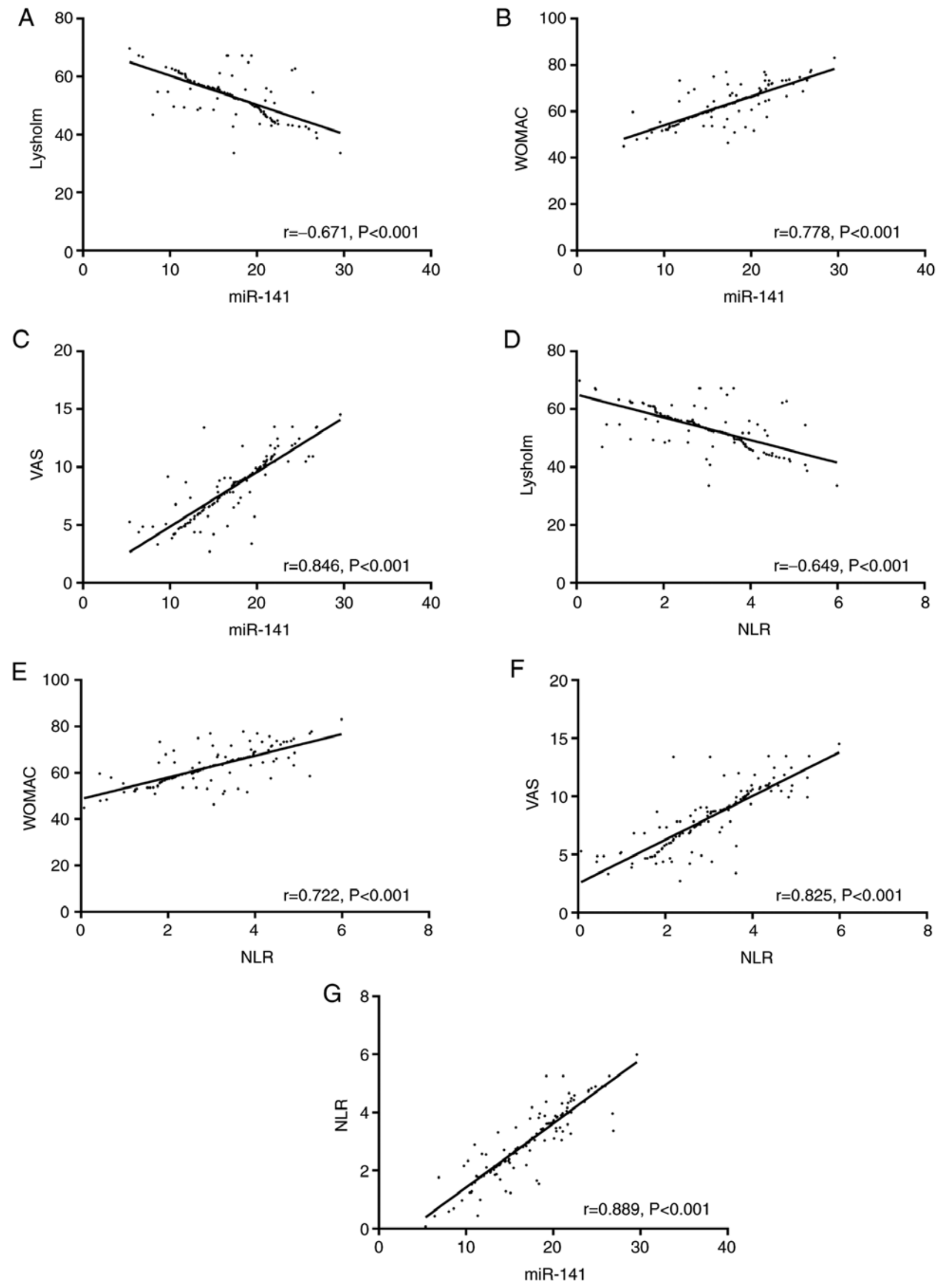

Figure 3. Correlations of miR-141 and NLR with LKSS, WOMAC, and VAS scores. (A) miR-141 was negatively correlated with LKSS score (r=-0.671, $\mathrm{P}<0.001)$. (B) miR-141 was positively correlated with WOMAC score $(\mathrm{r}=0.778, \mathrm{P}<0.001)$. (C) miR-141 was positively correlated with VAS score ( $\mathrm{r}=0.846$, $\mathrm{P}<0.001)$. (D) NLR was negatively correlated with LKSS score $(\mathrm{r}=-0.649, \mathrm{P}<0.001)$. (E) NLR was positively correlated with WOMAC score $(\mathrm{r}=0.722, \mathrm{P}<0.001)$. (F) NLR was positively correlated with VAS score $(r=0.825, \mathrm{P}<0.001)$. $(\mathrm{G})$ miR-141 was positively correlated with NLR $(\mathrm{r}=0.889$, $\mathrm{P}<0.001)$. miR, microRNA; NLR, neutrophil-lymphocyte ratio; LKSS, Lysholm Knee Scoring Scale; WOMAC, Western Ontario and McMaster Universities Osteoarthritis Index; VAS, visual analogue scale. 
Table VI. Assignment.

\begin{tabular}{|c|c|}
\hline Factors & Assignment \\
\hline Past medical history & $\begin{array}{l}\text { None }=0 ; \text { diabetes }=1 ; \\
\text { hypertension }=2\end{array}$ \\
\hline Exercise habits & Yes $=0 ; n o=1$ \\
\hline Course of disease & $\begin{array}{l}\text { A continuous variable analyzed by } \\
\text { original data }\end{array}$ \\
\hline Severity & $\begin{array}{l}\text { Early stage }=0 ; \text { middle and advanced } \\
\text { stages }=1\end{array}$ \\
\hline $\begin{array}{l}\text { Classification of } \\
\text { diseases }\end{array}$ & Primary $=0 ;$ secondary $=1$ \\
\hline miR-141 & $\begin{array}{l}\text { A continuous variable analyzed by } \\
\text { original data }\end{array}$ \\
\hline NLR & $\begin{array}{l}\text { A continuous variable analyzed by } \\
\text { original data }\end{array}$ \\
\hline
\end{tabular}

miR, microRNA; NLR, neutrophil-lymphocyte ratio.

(LR) was selected for multivariate logistic regression analysis. The results showed that the course of disease, severity, and the classification of diseases were not independent risk factors for osteoarthritis. However, past medical history (OR: 12.245; 95\% CI: 2.652-54.262), miR-141 (OR: 1.256; 95\% CI: 1.154-1.452), and NLR (OR: 25.627; 95\% CI: 1.953-86.621) were the independent risk factors. Exercise habits (OR: 0.497; 95\% CI: 0.085-3.215) was the independent protective factor for the disease (Table VII).

\section{Discussion}

As a chronic inflammation with articular cartilage degeneration as the core, osteoarthritis is mainly manifested as multi-directional and multi-level bone structural lesions (22). It causes arthralgia, joint dysfunction, and joint deformity, affecting the normal life and actions of patients (23). Therefore, a new clinical index for its early screening is being explored to effectively diagnose and treat this disease. For example, a study by Li et al suggested that ADAMTS-4 may be a potential marker for osteoarthritis (24). Park et al revealed that the detection of CTX-II in serum and urine by BeadChip had a markedly high diagnostic value for the disease (25). Previous studies have revealed that differences in miR expression are closely associated with cartilage destruction, chondrocyte apoptosis, and synovitis during the development and progression of certain osteoarticular diseases $(26,27)$. miR-141, which belongs to the miR-200 family and is located at $12 \mathrm{p} 13.31$ in the human body, was revealed to be closely related to gastric and non-small cell lung cancer $(28,29)$, however, its role in osteoarthritis is still unclear. In the present study, the roles of miR-141 and NLR in osteoarthritis were explored, and the findings indicated that they are essential for the diagnosis and treatment of the disease.

In the present study, miR-141 and NLR were significantly increased in the peripheral blood of patients with osteo- arthritis, suggesting that they may be involved in the development and progression of the disease. This is consistent with the findings of Dong et al (miR-141 in endometrial cancer) and Gundogdu et al (NLR in osteoarthritis) $(30,31)$, which support the results of the present study. The mechanism of action of miR-141 in osteoarthritis remains unclear. As an osteoblastic negative regulatory factor, miR-141 inhibits the cascade action of Dlx5/Runx2/Msx2 axis after transcription, thus reducing osteogenic activities (32). Karlsson et al revealed that the Msx 2 gene is involved in the bone formation of patients with osteoarthritis (33). Therefore, it is speculated that miR-141 may inhibit Msx2 and then inhibit the activation of osteoblasts, thereby deteriorating osteoarthritis. NLR, a blood inflammatory marker obtained through the ratio of neutrophils to lymphocytes, has a better predictive value for inflammation than traditional and single inflammatory markers (34). In our study, NLR in patients with osteoarthritis was significantly increased, suggesting that adverse conditions caused by inflammatory stimulation significantly reduce the lymphocytes of patients. The mechanism of NLR affecting the disease may be achieved through IL-6, IL-8, and TNF- $\alpha$ (35). A study by Goldring and Otero et al revealed the significant increase of the inflammatory cytokines in patients with osteoarthritis (36), which also confirms our theory. Inflammatory cytokines can enhance the cellular activity of the synovial tissue, thus neutrophils, platelets, or lymphocytes in peripheral blood significantly increase, while other indices are stable in patients with tumors and immune diseases (37). Therefore, NLR is commonly used as an indicator to evaluate the health of patients, however its diagnostic specificity for a certain disease is poor (38). In the present study, the combined detection of miR-141 and NLR had better sensitivity and specificity for predicting osteoarthritis. This reveals that the combined detection can be used for the early screening of osteoarthritis in clinical practice, and can improve its early diagnostic rate, which is important for the early treatment of the patients. At present, the diagnosis of osteoarthritis depends on imaging technology. The detection of miR-141 combined with NLR has several advantages. Firstly, the evaluation is objective. The assessment of results is not affected by the previous experience and subjective consciousness of doctors. Secondly, detection methods are simple. The peripheral blood is only needed for detection. Thirdly, the preservation time is long. The preservation time of peripheral blood in the low-temperature environment is long and the detection is less affected by environmental factors (39).

According to the analysis of the correlations of miR-141 and NLR with clinical pathology, miR-141 was closely associated to the course of disease, severity, and the classification of diseases. NLR was closely associated to age (40), past medical history, the course of disease, and severity. These results indicated that miR-141 and NLR are closely associated with osteoarthritis progression. There were differences in NLR between patients of different ages. This may be because the immune and metabolic functions of older patients decline, which makes them more vulnerable to inflammatory responses and diseases. Similarly, the findings of Soysal et al (41), support this theory. There were significant differences in the two indicators between patients with different classification 
Table VII. Multivariate logistic analysis.

\begin{tabular}{lccccrrr}
\hline & & & & & & \multicolumn{2}{c}{$95 \%$ CI } \\
\cline { 5 - 8 } Factors & $\mathrm{B}$ & $\mathrm{SE}$ & Wald & Sig & OR & Lower & Upper \\
\hline Past medical history & 2.524 & 0.862 & 9.412 & 0.002 & 12.245 & 2.652 & 54.262 \\
Exercise habits & 1.168 & 0.983 & 1.512 & 0.026 & 0.497 & 0.085 & 3.215 \\
miR-141 & 0.225 & 0.035 & 54.216 & $\mathrm{P}<0.001$ & 1.256 & 1.154 & 1.452 \\
NLR & 3.542 & 1.236 & 5.921 & 0.012 & 25.627 & 1.953 & 86.621 \\
\hline
\end{tabular}

miR, microRNA; NLR, neutrophil-lymphocyte ratio; B, regression coefficient; SE, standard error of regression; Wald, Wald statistics; df, degrees of freedom; Sig, P-value; OR, odds ratio.

of diseases, which may be due to the fact that patients with primary osteoarthritis usually have no trauma, infection, congenital malformation, genetic defects, and abnormalities in systemic metabolism and endocrine, with lighter conditions and lower treatment difficulty compared with those of patients with secondary arthritis (osteoarthritis occurring based on local original joint lesions). This is similar to the experimental results of Lunebourg et al (42). According to the correlations of miR-141 and NLR with LKSS, WOMAC, and VAS scores, miR-141 and NLR were negatively correlated with LKSS score, but positively correlated with WOMAC and VAS scores. This suggests that the two indicators are closely related to the conditions of patients with osteoarthritis. The two were also significantly correlated, which indicates that they have consistent synergy and may be closely related to the inflammatory responses of patients. According to the multivariate logistic regression analysis, past medical history, miR-141, and NLR were independent risk factors for osteoarthritis, demonstrating that miR-141 and NLR may be potential therapeutic targets for the disease. Exercise habits were the independent protective factor for the disease, suggesting that proper exercise can prevent its development, thus the importance of regular exercise should be emphasized for middle-aged people.

The present study aimed to explore the roles of miR-141 and NLR in osteoarthritis, but it has limitations due to the limited experimental conditions. For example, the underlying mechanisms of the effects of miR-141 and NLR in osteoarthritis are still conjecture and lack the support of basic experiments. We hope in future that relevant experimental analysis can be conducted according to our conjecture. In addition, the age range of the subjects included in the present study was small and the differences in regions and ethnicities was limited, therefore the results may be biased. Moreover, sequences of the miR family are numerous, thus there may be other miRs especially expressed in osteoarthritis, which require further analysis and discussion. In future, this study will be improved in order to achieve optimal experimental results.

In summary, miR-141 and NLR were significantly increased in the peripheral blood of patients with osteoarthritis. Their combined detection has a good diagnostic value for the disease and may become a potential therapeutic target for it in the future.

\section{Acknowledgements}

Not applicable.

\section{Funding}

The present study was supported by the National Natural Science Foundation of China: The research of Bi syndrome and body constitution as risk factors in predicting progression of osteoarthritis (funding project no. 81373665) and the Shanghai Science and Technology Commission Project: Optimization of TCM comprehensive diagnosis and treatment plan for senile Cervical Radiculopathy (funding project no. 18401901700).

\section{Availability of data and materials}

The datasets used and/or analyzed during the present study are available from the corresponding author on reasonable request.

\section{Authors' contributions}

RZ conceived the study and wrote the manuscript. CC performed the PCR experiments. XX analyzed and interpreted the data of patients. GL and HG helped with the statistical analysis. All authors read and approved the final manuscript.

\section{Ethics approval and consent to participate}

The present study was approved by the Ethics Committee of Shanghai TCM-Integrated Hospital, Shanghai University of TCM. Patients who participated in the present research, signed the informed consent and had complete clinical data. Signed written informed consents were obtained from the patients and/or guardians.

\section{Patient consent for publication}

Not applicable.

\section{Competing interests}

The authors declare that they have no competing interests. 


\section{References}

1. Malfait AM: Osteoarthritis year in review 2015: Biology. Osteoarthritis Cartilage 24: 21-26, 2016.

2. Loeser RF, Collins JA and Diekman BO: Ageing and the pathogenesis of osteoarthritis. Nat Rev Rheumatol 12: 412-420, 2016.

3. Nelson AE: Osteoarthritis year in review 2017: Clinical. Osteoarthritis Cartilage 26: 319-325, 2018.

4. Palazzo C, Nguyen C, Lefevre-Colau MM, Rannou F and Poiraudeau S: Risk factors and burden of osteoarthritis. Ann Phys Rehabil Med 59: 134-138, 2016.

5. Yu D, Jordan KP, Bedson J, Englund M, Blyth F, Turkiewicz A, Prieto-Alhambra D and Peat G: Population trends in the incidence and initial management of osteoarthritis: Age-period-cohort analysis of the clinical practice research Datalink, 1992-2013. Rheumatology (Oxford) 56: 1902-1917, 2017.

6. Mathiessen A and Conaghan PG: Synovitis in osteoarthritis: Current understanding with therapeutic implications. Arthritis Res Ther 19: 18, 2017.

7. Sharma L: Osteoarthritis year in review 2015: Clinical. Osteoarthritis Cartilage 24: 36-48, 2016.

8. Collins NJ, Prinsen CA, Christensen R, Bartels EM, Terwee CB and Roos EM: Knee injury and osteoarthritis outcome score (KOOS): Systematic review and meta-analysis of measurement properties. Osteoarthritis Cartilage 24: 1317-1329, 2016

9. Wallace IJ, Worthington S, Felson DT, urmain RD, Wren KT, Maijanen H, Woods RJ and Lieberman DE: Knee osteoarthritis has doubled in prevalence since the mid-20th century. Proc Nat Acad Sci USA 114: 9332-9336, 2017.

10. Wang Y, Teichtahl AJ and Cicuttini FM: Osteoarthritis year in review 2015: Imaging. Osteoarthritis Cartilage 24: 49-57, 2016.

11. Rupaimoole R and Slack FJ: MicroRNA therapeutics: Towards a new era for the management of cancer and other diseases. Nat Rev Drug Discov 16: 203-222, 2017.

12. Feinberg MW and Moore KJ: MicroRNA regulation of atherosclerosis. Circ Res 118: 703-720, 2016.

13. Yang S, Zhang W, Cai M, Zhang Y, Jin F, Yan S, Baloch Z, Fang Z, Xue S, Tang R, et al: Suppression of bone resorption by miR-141 in aged rhesus monkeys. J Bone Miner Res 33: 1799-1812, 2018.

14. Hu H, Zhang G, Tian G, Lv G and Jin Y: miRNA profiling reveals the upregulation of Osteogenesis-associated miRNAs in ovariectomy osteoporosis mice. Clin Exp Obstet Gynecol 45: 817-822, 2018.

15. Balta S, Celik T, Mikhailidis DP, Ozturk C, Demirkol S, Aparci $\mathrm{M}$ and Iyisoy A: The relation between atherosclerosis and the neutrophil-lymphocyte ratio. Clin Appl Thromb Hemost 22: 405-411, 2016

16. Fu X, Li T, Dai Y and Li J: Preoperative systemic inflammation score (SIS) is superior to neutrophil to lymphocyte ratio (NLR) as a predicting indicator in patients with esophageal squamous cell carcinoma. BMC Cancer 19: 721, 2019.

17. Glyn-Jones S, Palmer AJ, Agricola R, Price AJ, Vincent TL, Weinans H and Carr AJ: Osteoarthritis. Lancet 386: 376-387, 2015.

18. Tichopad A, Kitchen R, Riedmaier I, Becker C, Ståhlberg A and Kubista M: Design and optimization of reverse-transcription quantitative PCR experiments. Clin Chem 55: 1816-1823, 2009.

19. Lysholm $\mathrm{J}$ and Gillquist J: Evaluation of knee ligament surgery results with special emphasis on use of a scoring scale. Am J Sports Med 10: 150-154, 1982.

20. McConnell S, Kolopack P and Davis AM: The western ontario and mcmaster universities osteoarthritis index (WOMAC): A review of its utility and measurement Properties. Arthritis Rheum 45: 453-461, 2001.

21. Wewers ME and Lowe NK: A critical review of visual analogue scales in the measurement of clinical phenomena. Res Nurs Health 13: 227-236, 1990.

22. Johnson VL and Hunter DJ: The epidemiology of osteoarthritis Best Pract Res Clin Rheumatol 28: 5-15, 2014.

23. Thakur M, Dickenson AH and Baron R: Osteoarthritis pain: Nociceptive or neuropathic? Nat Rev Rheumatol 10: 374, 2014.

24. Li W, Du C, Wang H and Zhang C: Increased serum ADAMTS-4 in knee osteoarthritis: A potential indicator for the diagnosis of osteoarthritis in early stages. Genet Mol Res 13: 9642-9649, 2014.
25. Park YM, Kim SJ, Lee KJ, Yang SS, Min BH and Yoon HC: Detection of CTX-II in serum and urine to diagnose osteoarthritis by using a Fluoro-microbeads guiding chip. Biosens Bioelectron 67: 192-199, 2015.

26. Li D, Liu J, Guo B, Liang C, Dang L, Lu C, He X, Cheung HY, $\mathrm{Xu} \mathrm{L}, \mathrm{Lu} \mathrm{C}$, et al: Osteoclast-derived exosomal miR-214-3p inhibits osteoblastic bone formation. Nat Commun 7: 10872, 2016.

27. Wu Y, Zhang Y, Zhang $\mathrm{Y}$ and Wang JJ: CircRNA hsa circ 0005105 upregulates NAMPT expression and promotes chondrocyte extracellular matrix degradation by sponging miR-26a. Cell Biol Int 41: 1283-1289, 2017.

28. Zhou X, Ye F, Yin C, Zhuang Y, Yue G and Zhang G: The interaction between MiR-141 and lncRNA-H19 in regulating cell proliferation and migration in gastric cancer. Cell Physiol Biochem 36: 1440-1452, 2015.

29. Tejero R, Navarro A, Campayo M, Viñolas N, Marrades RM, Cordeiro A, Ruíz-Martínez M, Santasusagna S, Molins L, Ramirez J, et al: miR-141 and miR-200c as markers of overall survival in early stage non-small cell lung cancer adenocarcinoma. PLoS One 9: e101899, 2014.

30. Dong Y, Si JW, Li WT, Liang L, Zhao J, Zhou M, Li D and Li T: miR-200a/miR-141 and miR-205 upregulation might be associated with hormone receptor status and prognosis in endometrial carcinomas. Int J Clin Exp Pathol 8: 2864-2875, 2015.

31. Gundogdu G and Gundogdu K: A novel biomarker in patients with knee osteoarthritis: Adropin. Clin Rheumatol 37: 2179-2186, 2018.

32. Sangani R, Periyasamy-Thandavan S, Kolhe R, Bhattacharyya MH, Chutkan N, Hunter M, Isales C, Hamrick M, Hill WD and Fulzele S: MicroRNAs-141 and 200a regulate the SVCT2 transporter in bone marrow stromal cells. Mol Cell Endocrinol 410: 19-26, 2015.

33. Karlsson C, Dehne T, Lindahl A, Brittberg M, Pruss A, Sittinger $\mathrm{M}$ and Ringe J: Genome-wide expression profiling reveals new candidate genes associated with osteoarthritis. Osteoarthritis Cartilage 18: 581-592, 2010.

34. Sunbul M, Gerin F, Durmus E, Kivrak T, Sari I, Tigen K and Cincin A: Neutrophil to lymphocyte and platelet to lymphocyte ratio in patients with dipper versus non-dipper hypertension. Clin Exp Hypertens 36: 217-221, 2014

35. Han R, Wu D, Deng S, Liu T, Zhang T and Xu Y: NLRP3 inflammasome induces pyroptosis in lung tissues of radiation-induced lung injury in mice. Xi Bao Yu Fen Zi Mian Yi Xue Za Zhi 33: 1206-1211, 2017 (In Chinese).

36. Goldring MB and Otero M: Inflammation in osteoarthritis. Curr Opin Rheumatol 23: 471-478, 2011.

37. Adamski JK, Kelsey A and Brennan B: Inflammatory myofibroblastic tumors following the treatment of malignancy in Childhood: Case reports. J Pediatr Hematol Oncol 36: 159-162, 2014.

38. Diaz-Martinez J, Campa A, Delgado-Enciso I, Hain D, George F, Huffman F and Baum M: The relationship of blood neutrophil-to-lymphocyte ratio with nutrition markers and health outcomes in hemodialysis patients. Int Urol Nephrol 51: 1239-1247, 2019.

39. Koolwal D: Anticoagulant EDTA induced storage effect (Artifacts) on peripheral blood cells. Clin Lab Haematol 27: 336-342, 2018

40. Niazi S, Krogh Nielsen M, Sørensen TL and Subhi Y: Neutrophil-to-lymphocyte ratio in age-related macular degeneration: A systematic review and meta-analysis. Acta Ophthalmol 97: 558-566, 2019.

41. Soysal P, Stubbs B, Lucato P, Luchini C, Solmi M, Peluso R, Sergi G, Isik AT, Manzato E, Maggi S, et al: Inflammation and frailty in the elderly: A systematic review and meta-analysis. Ageing Res Rev 31: 1-8, 2016.

42. Lunebourg A, Parratte S, Gay A, Ollivier M, Garcia-Parra K and Argenson JN: Lower function, quality of life, and survival rate after total knee arthroplasty for posttraumatic arthritis than for primary arthritis. Acta Orthop 86: 189-194, 2015.

This work is licensed under a Creative Commons Attribution-NonCommercial-NoDerivatives 4.0 International (CC BY-NC-ND 4.0) License. 